\title{
Tickling Expectations: Neural Processing in Anticipation of a Sensory Stimulus
}

\author{
Katrina Carlsson, Predrag Petrovic, Stefan Skare, \\ Karl Magnus Petersson, and Martin Ingvar
}

Karolinska Institute/Karolinska Hospital, Sweden

\begin{abstract}
- Predictions of the near future can optimize the accuracy and speed of sensory processing as well as of behavioral responses. Previous experience and contextual cues are essential elements in the generation of a subjective prediction. Using a blocked fMRI paradigm, we investigated the pattern of neural activation in anticipation of a sensory stimulus and during the processing of the somatosensory stimulus itself. Tickling was chosen as the somatosensory stimulus rather than simple touch in order to increase the probability to get a high degree of anticipation. The location and nature of the stimulus were well defined to the subject. The state of anticipation was initiated by attributing an uncertainty regarding the time of stimulus onset. The network of activation and deactivation during anticipation of the expected stimulus was similar to that engaged during the actual sensory stimulation. The areas that were activated
\end{abstract}

\section{INTRODUCTION}

The human capacity to perform real time information processing is limited. The limited speed of neuronal operations may partly be compensated for by parallel interactive and distributed processing (Rumelhart \& McClelland, 1986). Another mechanism may be the transformation of initially controlled processing towards automaticity. Automaticity implies a gain of speed and precision at the possible cost of flexibility (Schnieder \& Shiffrin, 1977). It also implies a decreased computational demand, which in turn improves the capacity for simultaneous processing. An alternative way of increasing the efficiency of information processing is to continuously update predictions of what is to be expected. Explicit or implicit predictions allow for selective and focussed processing of sensory input (Corbetta, Miezin, Dobmeyer, Shulman, \& Petersen, 1991; Posner \& Petersen, 1990) as well as detailed planning of behavior and a reduction of the number of options in the behavioral repertoire (Broadbent, 1958). If a subject is able to make accurate predictions, the time and accuracy of responses at the behavioral level are improved (Posner during both states included the contralateral primary sensory cortex, bilateral areas in the inferior parietal lobules, the putative area SII, the right anterior cingulate cortex and areas in the right prefrontal cortex. Similarly, common decreases were observed in areas of sensorimotor cortex located outside the area representing the target of stimulus, i.e., areas that process information which is irrelevant to the attended process. The overlapping pattern of change, during the somatosensory stimulation and the anticipation, furthers the idea that predictions are subserved by a neuronal network similar to that which subserves the processing of actual sensory input. Moreover, this study indicates that activation of primary somatosensory cortex can be obtained without intra-modal sensory input. These findings suggest that anticipation may invoke a tonic top-down regulation of neural activity.
\& Petersen, 1990). Hence, anticipation allows for more pertinent reactions in the immediate situation (James, 1890).

A high-quality prediction in the somatosensory domain carries defined information, not only on what or where something is going to happen, but also when it will happen. The most accurate predictions can be made when the input represents sensory feedback from self-generated actions since this implies precise information on all aspects including the time domain of the coming event (Blakemore, Wolpert \& Frith, 1999; Blakemore, Wolpert, \& Frith, 1998). The predictions become more uncertain when they are applied to externally generated sensory input. Previously acquired knowledge, combined with presently available contextual information from different sensory modalities may then be used to generate predictions. For example, a prediction of the tactile aspects of a planned touch of a surface may be made from visual input in combination with experience. A manipulation of the degree of predictability of an event provides an opportunity to study anticipatory mechanisms in the brain (Hsieh, Stone-Elander, \& Ingvar, 1999). 
Conceptually, relevant predictions may serve as a guide for attention. In experimental settings, it has been shown that attention mechanisms may modulate the activity in lower order sensory cortices (Frith \& Dolan, 1997; Drevets et al., 1995; Corbetta et al., 1991). In the study by Drevets et al. (1995), the subjects were asked to count infrequent touches with von Frey's hair or anticipate a painful stimulus. That PET study showed decreased neural activity in the parts of the primary sensory cortex that were outside the area representing the skin locus of the expected stimuli. In an earlier 2-D rCBF ${ }^{133}$ Xe study, with a similar task of counting infrequent light touches, increased neural activity was reported in an area of primary sensory cortex defined as representing the target of stimulation (Roland, 1981). In spite of the apparent inconsistency between those results, possibly due to methodological differences, they both point to the possible existence of an attentional modulation during anticipation. The attentional mechanism for such a modulation of neural activity in the absence of stimulus presentation can be described in terms of a bias signal (Rees \& Frith, 1998). A bias signal would improve the computational efficiency in the area of interest and suppress responses outside the focus of attention (Ghatan, Hsieh, Petersson, Stone-Elander, \& Ingvar, 1998). Modulations of relevant low level processing areas without external input have also been illustrated in motor and sensory imagery studies, i.e., in a situation when the subject is imagining the stimulus, but not expecting a real stimulus in the near future (Hodge, Dubroff, Huckins, \& Szeverenyi, 1998; Kosslyn, Thompson, \& Alpert, 1997; Roth et al., 1996). The activation of the same neural network during imagery as that which is responsible for the actual movement or sensation is consistent with the suggestion by James (1890) that "sensation and imagination are due to the activity of the same centers in the cortex."

The underlying hypothesis for the present work is that a prediction of an external event is a cerebral mechanism that serves to optimize the accuracy and speed of sensory perception. Based on earlier imaging data (e.g., Drevets et al., 1995; Roland, 1981) we designed an experiment to test the hypothesis that neuronal computations may precede the actual response or behavior. Specifically, we wanted to test if the neural response in anticipation of a sensory input is similar to the response to the actual sensory input. Tickle is considered to be a more potent somatosensory stimulus than ordinary touch. In order to increase the probability of getting a strong anticipatory response, we therefore chose tickling instead of touch as the stimulus. The sensory stimulation of tickle is unique in several respects. To denote a sensation as tickle, the touch must be light and the tickled part is preferably a body part not commonly touched. It is also perceived as more intense if another person induces the tickling (Blakemore, Wolpert, et al., 1998; Mintz, 1967). Consequently, we tickled the subjects with a light touch of painter's brush on the foot sole. A similarity between the patterns of response during anticipation and tickling would further the idea that predictions are subserved by a similar neuronal network as that which subserves the processing of actual sensory input. We used functional magnetic resonance imaging (fMRI) to monitor the functional activity during anticipation of a well-defined somatosensory stimulus as well as during the sensory stimulus itself. The anticipatory state was elicited with a visual cue that carried information of a change in context and thereby indicated when the subject should anticipate a sensory stimulus.

\section{RESULTS}

All subjects withstood the sessions in the camera well and none complained of discomfort. Before entering the camera, the subjects rated their level of ticklishness, The self-reported estimation was $66 \pm 6$ (mean \pm SEM) on the VAS scale (0-100, not at all ticklish-more ticklish than anyone I know). The subjects estimated the mean tickling sensation in the experimental situation to be 50 \pm 6 (0-100, no experience of tickle-maximally imaginable level of tickling) and the desire to move during tickle to $40 \pm 8$ (0-100, no desire to move-irresistible desire to move). None of the subjects described the tickling as unpleasant. All subjects associated the red color square with tickling, described increased alertness when it was displayed, and acknowledged a sense of anticipation during the runs when the red square was shown with no tickling. In four out of seven cases, the sense of anticipation was reported as a marked phenomenon. The sensation of tickle was perceived as more intense at the end of each tickling epoch (4/7 cases).

\section{Sensory Stimulation Compared to Rest (SS versus RS)}

Maximum $Z$ scores for MRI signal change in the sensory stimulation state versus rest are listed in Table 1A. Activated areas in the right frontal lobe, ipsilateral to the stimuli, included parts of orbitofrontal and lateral frontal cortex (Broddman's area (BA) 10, 44, 47 and 49) as well as the anterior cingulate (ACC, BA 32). In the left frontal lobe, the activations were restricted to BA 44. Increases were also observed in the parietal lobe in the postcentral gyrus bilaterally (SI, BA 1-3) in a location that was consistent with the sensory area representing the foot, bilaterally in the inferior parietal lobule and in areas presumably approximating SII. On the contralateral side this activation had its center in BA 43/40 of the parietal operculum and extended into adjacent parts of the postcentral gyrus. On the right side the activated area was seen somewhat more posterior in BA 40 located mostly behind, but also spreading into the opercular region (Figure 1). 
Table 1. Maximum $Z$ Scores and Cluster Size Obtained in a Voxel-by-Voxel Comparison for Main Effects Contrasts

Region/structure

(A) Tickle versus rest (SS versus RS)

Prefrontal activations

Superior frontal gyrus

Inferior frontal gyrus

Cingulate activations

Anterior cingulate

Parietal activations

Postcentral gyrus

Inferior parietal lobule

Prefrontal acitvations

Middle frontal gyrus

Inferior frontal gyrus

Sensorimotor activations

Pre- and postcentral gyrus

Cingulate activations

Midline midcingulate

Midline posterior cingulate

Parietal activations

Superior parietal lobule

Inferior parietal lobule

Midline parietal lobule
BA 10 right

9, 5.15

BA $44 * / 47 / 49 *$ right

BA $44^{*}$ left

BA 32 right

$3,5.04$

.005

4, 16, 40

$112,7.53$

$15,6.82$

$<.001$

$<.001$

$16,60,16$

40, 20, 0

$-60,4,4$

4, 5.39

$10,6.74$

$18,7.10$

$3,7.27$

$23,7.24$

2, 4.79

60, 7.80

$88,8.02$

BA 40* right

BA 40/43* left

BA 40 right

BA 40 right

$$
\begin{array}{r}
.001 \\
<.001 \\
<.001 \\
<.001
\end{array}
$$

$<.001$

.013

$<.001$

$<.001$
$20,-40,68$

$12,-48,68$

$-24,-36,68$

$-32,-40,64$

$56,-24,24$

$44,-32,32$

$60,-48,12$

$-60,-20,16$
BA 8/9 right

BA 10 left

BA 10 left

BA 8 left

BA 6/8 left

BA 44 left

BA 1-3/4 right

BA $1-3 / 4$ left

BA $24 * / 32 *$

BA $23 * / 31 *$
$37,6.46$

$16,6.53$

7, 4.55

4, 5.21

49, 7.13

23, 6.59

$127,7.60$

$123,7.25$

$31,7.27$

140, 6.85
$<.001$

$<.001$

.032

.002

$<.001$

$<.001$

$<.001$

$<.001$

$36,-20,40$

$-40,20,44$
$-32,60,4$

$-36,44,-8$

$-48,20,36$

$-28,16,48$

$-40,20,24$
BA $7 / 39 * / 19$ right

BA 39 left

BA 40 left

BA 7
$18,4.77$

$8,6.35$

3, 4.63

$11,5.67$
$<.001$

$<.001$
$0,-24,48$

$-8,-68,28$

\begin{tabular}{rl}
.004 & $40,-64,40$ \\
$<.001$ & $-36,-52,20$ \\
.024 & $-36,-56,44$ \\
$<.001$ & $-4,-68,48$ \\
\hline
\end{tabular}

(continued) 
Table 1. (continued)

\begin{tabular}{|c|c|c|c|c|}
\hline Region/structure & $B A$ & $k, z$ value & p value & $x, y, z$ \\
\hline \multicolumn{5}{|c|}{ (C) Anticipation versus rest (AS versus RS) } \\
\hline \multicolumn{5}{|l|}{ Prefrontal activations } \\
\hline \multirow[t]{2}{*}{ Inferior frontal gyrus / Insula } & $\begin{array}{l}\text { BA 47/49*/44/45/ } \\
14 / 15 \text { right }\end{array}$ & $80,7.28$ & $<.001$ & $36,24,-4$ \\
\hline & BA 47 left & $11,6.35$ & $<.001$ & $-32,28,-4$ \\
\hline \multicolumn{5}{|l|}{ Cingulate activations } \\
\hline Anterior cingulate & BA 32 right & $5,5.91$ & $<.001$ & $4,16,40$ \\
\hline \multicolumn{5}{|l|}{ Parietal activations } \\
\hline Postcentral gyrus & BA $1-3 *$ left & $8,5.77$ & $<.001$ & $-20,-44,60$ \\
\hline Inferior parietal lobule & $\begin{array}{l}\text { BA } 40 \text { right } \\
\text { BA } 40 * \text { right } \\
\text { BA } 40 / 43 * \text { left }\end{array}$ & $\begin{array}{l}32,5.47 \\
44,7.38 \\
3,4.55\end{array}$ & $\begin{array}{r}<.001 \\
<.001 \\
.033\end{array}$ & $\begin{array}{l}48,-44,48 \\
52,-44,20 \\
-52,-32,24\end{array}$ \\
\hline Superior parietal lobule & BA 7 right & $11,5.67$ & $<.001$ & $-4,-68,48$ \\
\hline
\end{tabular}

(D) Rest versus anticipation (RS versus AS)

Prefrontal activations

Middle frontal gyrus

Sensorimotor activations

Pre- and postcentral gyrus

Cingulate cortex

Midline midcingulate

Midline posterior cingulate
BA $24 * / 32 *$

BA $23 * / 31 *$

$4,4.54$

$43,6.54$

BA 8 left

BA 1-3/4 right

BA 1-3/4 left

$73,7.05$

$81,5.97$

$57,7.14$

$67,6.03$
$<.001$

$<.001$
24, 20, 36

$-28,24,44$
$<.001$

$<.001$

$28,-28,64$

$-24,-24,56$

\section{(E) Tickle versus anticipation (SS versus AS)}

Frontal activations

Inferior frontal gyrus / Insula

BA 14/15/44* right

BA $44^{*}$ left

14/15 left

$19,5.38$

$5,5.02$

$3,4.94$

.004

.005

$19,5.54$

$14,5.16$

$73,7.65$

$4,5.12$

$7,5.61$

$8,5.43$

$3,5.05$

$$
\begin{array}{r}
<.001 \\
.003 \\
<.001 \\
\\
.003 \\
<.001 \\
.001 \\
.004
\end{array}
$$

$60,-24,24$

$60,-48,12$

$-64,-20,12$

$24,-40,68$

$12,-48,68$

$-24,-36,68$

$-32,-40,64$

(continued) 
Table 1. (continued)

\begin{tabular}{|c|c|c|c|c|}
\hline Region/structure & $B A$ & $k, z$ value & p value & $x, y, z$ \\
\hline \multicolumn{5}{|c|}{ (F) Anticipation versus tickle (AS versus SS) } \\
\hline \multicolumn{5}{|l|}{ Frontal activations } \\
\hline Middle frontal gyrus & BA 10 left & $14,6.26$ & $<.001$ & $-32,56,4$ \\
\hline Middle/inferior frontal gyrus & $\begin{array}{l}\text { BA } 11 / 47 \text { left } \\
\text { BA } 9 / 46 \text { left }\end{array}$ & $\begin{array}{l}16,4.70 \\
19,5.33\end{array}$ & $\begin{array}{l}.006 \\
.001\end{array}$ & $\begin{array}{l}-36,40,-4 \\
-48,32,24\end{array}$ \\
\hline \multicolumn{5}{|l|}{ Sensorimotor activations } \\
\hline Pre- and postcentral gyrus & $\begin{array}{l}\text { BA 1-3/4 right } \\
\text { BA 1-3/4 left }\end{array}$ & $\begin{array}{l}18,5.66 \\
18,5.06\end{array}$ & $\begin{array}{r}<.001 \\
.004\end{array}$ & $\begin{array}{l}36,-20,40 \\
-32,-20,40\end{array}$ \\
\hline \multicolumn{5}{|l|}{ Cingulate cortex } \\
\hline Midline posterior cingulate & $\begin{array}{l}\text { BA } 31 \\
\text { BA } 31\end{array}$ & $\begin{array}{l}2,4.51 \\
25,4.82\end{array}$ & $\begin{array}{l}.037 \\
.001\end{array}$ & $\begin{array}{l}16,-44,32 \\
-12,-64,32\end{array}$ \\
\hline \multicolumn{5}{|l|}{ Parietal activations } \\
\hline Superior parietal lobule & BA 7 right & $14,4.76$ & .009 & $28,-64,44$ \\
\hline
\end{tabular}

\section{Decreases of Activity During Sensory Stimulation (RS versus SS)}

Decreased activity compared to rest (Table 1B) was observed bilaterally in parts of the primary sensorimotor cortex (SMC, BA 1-3/4) that were located outside the area representing the foot. Also, parts of prefrontal cortex (PFC) showed deactivation, more extensively on the side lobe (BA 6/8, 10, 44), but also on the right (BA 8, 9). Other areas showing significant deactivations were the side midcingulate (BA 24/32), posterior cingulate bilaterally (BA 23/31), superior and inferior parietal lobe bilaterally (BA 7/19/39/40).

\section{Anticipation Compared to Rest (AS versus RS)}

As shown in part $\mathrm{C}$ of Table 1 a similar network as that in SS versus RS was activated by anticipation. Activations in the right PFC overlapped considerably with those activated in the SS with increases in BA 44, 45, 47, 49 and the ACC, BA 32. Additionally, the right insula was activated. In the left frontal lobe, an activation in BA 47 was noted. A striking finding was the activation confined to the contralateral SI complementary to the activation seen in SS. Also, the inferior parietal lobe activation described above in the SS versus RS, was found. Thus, on the contralateral side the activation was found in the parietal operculum (BA 43) and on the ipsilateral side the activation was situated behind the operculum, located in BA 40. These activations were less extensive, especially in the left hemisphere, than in the corresponding SS versus RS contrast. Additionally, an activation was seen in precuneus (BA 7).

\section{Decreases of Activity During Anticipation (RS versus AS)}

Also the deactivations during anticipation (part D, Table 1) showed distinct similarities to the deactivations during tickle. The bilateral deactivations in the frontal eyefield (BA 8) were present along with the bilateral deactivation in SMC (BA 1-3/4), the midcingulate and the posterior cingulate deactivation (BA 23/31).

\section{Increases and Decreases in the Comparison of Anticipation and Tickle (SS versus AS) and (AS versus SS)}

The contrast SS versus AS showed a similar pattern of response compared to SS versus RS. In SS versus AS (part E, Table 1) the activations were bilaterally located in the lateral frontal cortices (BA 44), in the insulae in the SI areas (BA 1-3) and in the inferior parietal lobes (BA 40/43).

Correspondingly, the contrast AS versus SS was comparable to the contrast RS versus SS. In AS versus 


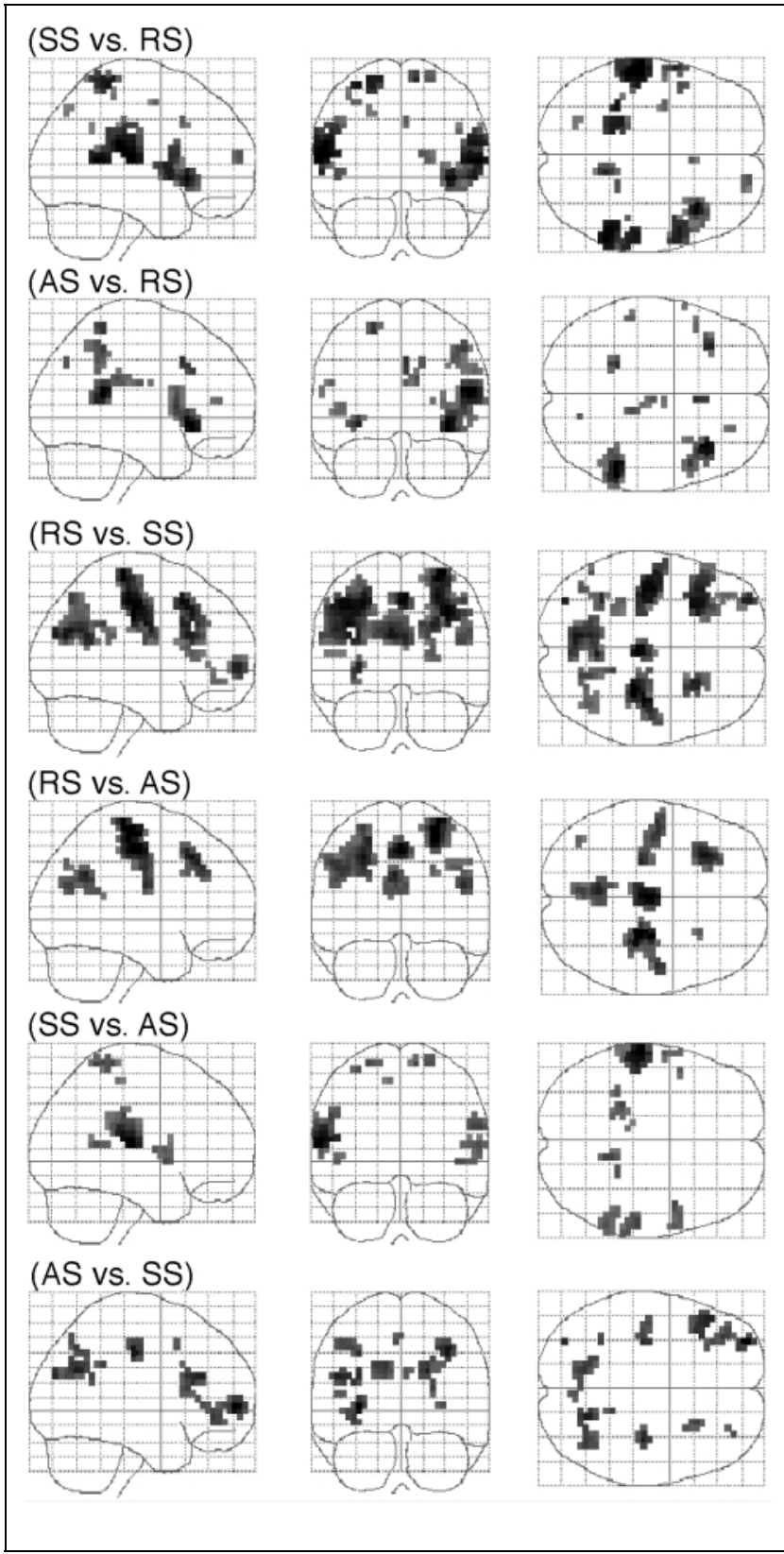

Figure 1. Sagittal, coronal and axial views of maximum-intensity projections of statistical parametric maps showing significant activations in tickle versus rest (SS versus RS), anticipation versus rest (AS versus RS), rest versus tickle (RS versus SS), rest versus anticipation (RS versus AS), tickle versus anticipation (SS versus AS) and anticipation versus tickle (AS versus SS). All images are thresholded at the omnibus significance level of $p=.0001(z=3.72)$.

SS (part F, Table 1), there were significant clusters in the left PFC (BA 10, 11/47, 9/46) in the SMC bilaterally (BA 1-3/4), in the posterior cingulate cortex (BA 31) and in the superior parietal lobe (BA 7).

\section{Time Characteristics of the AS Response}

In Figure 2, the time-activity curve was plotted for a coordinate in SI from the subset of sessions where AS was presented without a following SS in the last two blocks. The first peak reflects three time-points of AS immediately followed by three representing SS. The two subsequent responses represent data from six time-points of AS with no sensory stimulus. Thus, the anticipatory response in SI was present also when the anticipatory state was not followed by sensory stimulation.

\section{DISCUSSION}

The main finding of this fMRI study was that the overall response to anticipation had a strikingly similar pattern to the response of the actual sensory stimulation, and that the anticipation state and the sensory stimulation state both elicited responses in the primary and secondary sensory cortices.

Additional to the unique characteristics of the sensory stimulation of tickle, which may for example evoke excitation and arousal, there is a resemblance to other sensations like touch and vibration and sometimes even itch and slight pain (Mintz, 1967). Tickle, itch, and pain all hold affective components and also to give rise to a motor intention to move away (pain, tickle) or towards (itch) the stimulus (Hsieh, 1995).

The subjects in the present experiment were not required to give any response and were explicitly instructed to not move during scanning. Part of the reported activations may therefore be explained by the control of an urge to move (withdrawal of the limb) during the sensory stimulation. In Hsieh et al. (1994) this was specifically addressed by injecting an itch-inducing agent (histamine). The subjects felt an urge to move but remained still according to the instructions to not move. The activated regions reported in Hsieh et al. (1994) showed a limited similarity with our present data. For example, the prominent activation in the right dorsolateral prefrontal cortex (BA 46) was not replicated in the present study. Some of the activations were, however, replicated in this study, including the right inferior parietal lobule. In the previous communication, it was concluded that the urge to scratch was expressed as a pattern of activation partly corresponding to the inhibition of a planned complex motor response. The potential motor response to tickling in this study is supposedly less complex entailing only a simple withdrawal of the limb. Therefore, we suggest that the motor planning component is restricted to the inhibition of a stereotype response. This may explain the differences of results related to regions subserving complex motor responses between the present data and Hsieh et al. (1994).

The limited capacity of the brain for on-line processing makes it of value to predict upcoming events (Ingvar, 1985). A computationally effective mode for the generation of a prediction is the use of an internal model. Previously acquired knowledge in combination with contextual information generates the basis for such model-based predictions. This can be an effective 
Figure 2. The relation between mean adjusted response (i.e., adjusted for effects of no interest, arbitrary units) and time are plotted for a coordinate in area SI $(x=-20, y=$ $-44, z=60)$. Figure represents the 19 series of scans from seven subjects that used the protocol (RS (10), AS (3), SS (3), RS (10), AS (6), RS (10), AS (6), RS (10) $=58$ images). RS $=$ empty circles; AS = filled triangles; SS = filled squares.

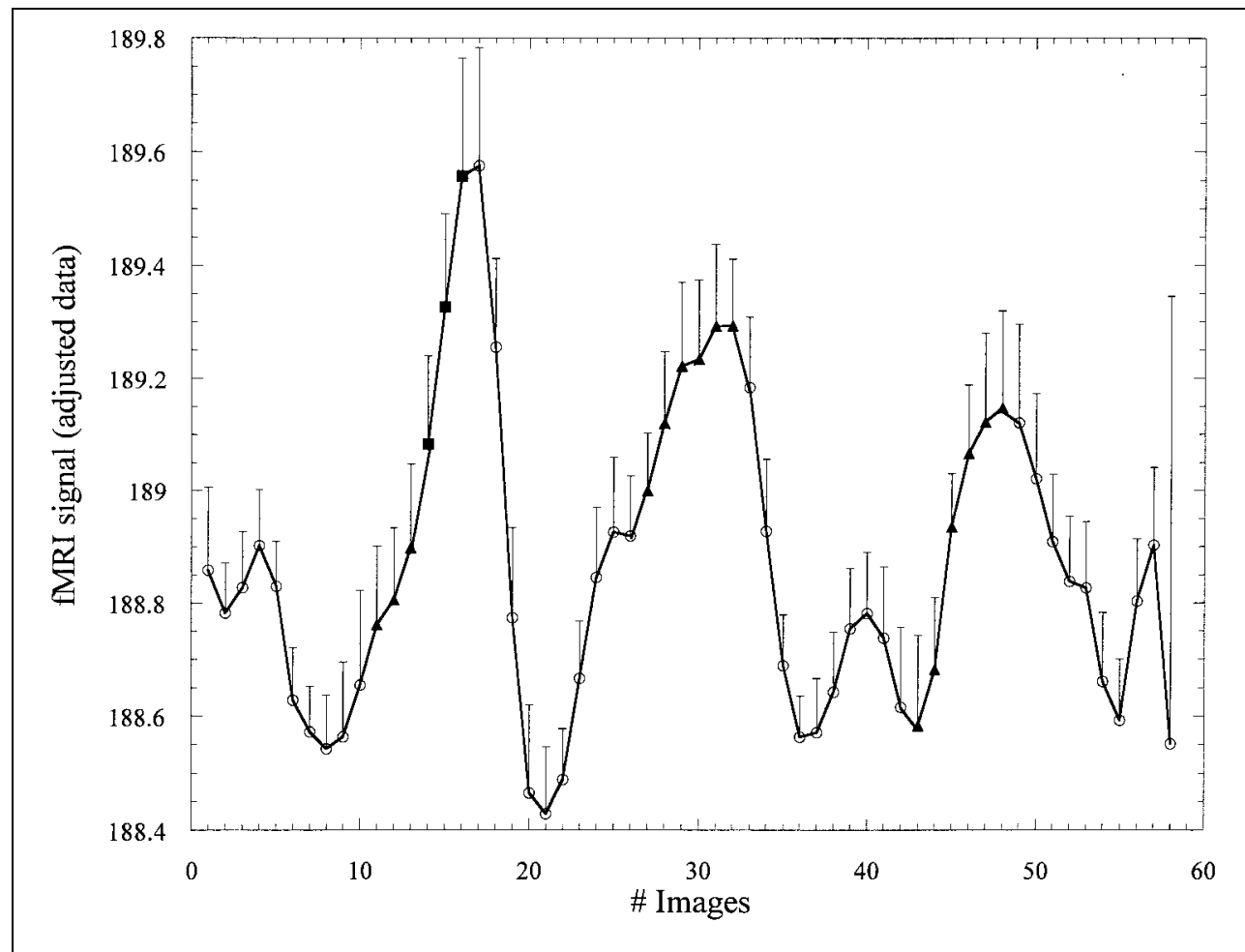

way of making appropriate selections from the repertoire of perceptions and behaviors. The use of expectations yields information on sensory input regarding timing, modality, and even the nature of stimulus within a modality. This mechanism may explain why the perception of an expected sensory event is different from an unexpected event.

A special aspect of prediction is when a sensory event is a consequence of a self-generated action (Blakemore, Wolpert, et al., 1998). It has been hypothesized that self-generated models for actions, generate not only the action as such but also a prediction of the sensory feedback of the same action (for a review see Wolpert, 1997). Since movements usually require a more rapid sensory feedback than the relatively slow sensorimotor loops allow, a preestimation for a sensory outcome provided by an internal model can supply approximately correct information during the feedback delay. In this way, the expected and actual sensory outcome can be compared to generate suitable correction/learning signals. Similarly, an internal simulation of a movement can be used in mental practice (Wolpert, 1997). The "efference copy" of the motor output can also be used to filter out self-induced sensory feedback, thereby contributing to the ability to distinguish between signals originating from self-generated actions and the environment (Wolpert, 1997; Gallistel, 1980; Von Holst, 1954). The resulting attenuation of incoming signal from self-generated movement has been demonstrated both for proprioceptive input
(Weiller et al., 1996) as well as for sensory stimulation by tickling or tones (Blakemore, Rees \& Frith, 1998; Blakemore, Wolpert, et al., 1998). When performing a self-initiated action, the subject is in control of the sensory domain, both regarding time and space. The prediction of the sensory input is therefore of high quality and the activation of the sensory cortices is moderate. When the stimulus is externally induced, the subject has less control of the sensory domain. Then, the quality of the prediction is lower and any sensory stimulus will generate a more expressed response in the sensory cortices (Blakemore, Rees, et al., 1998; Blakemore, Wolpert, et al., 1998; Weiller et al., 1996). The internal "forward model" may contribute to the ability to identify the signals that are a result of our own actions. Internally generated predictions may also be one mean, whereby the need for intensive on-line computations is decreased. In this paper, we extended this line of reasoning by studying how external contextual information given beforehand, may allow for prediction of a sensory percept. The experimental paradigm allowed us to study how this anticipation is processed in itself.

The results indicate that the pattern of activations during tickle (SS versus RS) and anticipation (AS versus RS) were similar, consistent with our initial hypothesis. The similarity of the responses indicates the existence of internal mechanisms for generating a prediction regarding a sensory percept based solely on a change in contextual information. The main areas that were 
activated in common during tickle (SS versus RS) and anticipation (AS versus RS) included contralateral primary sensory cortex representing the foot, bilateral areas in the inferior parietal lobules, areas probably approximating SII, right (ipsilateral) anterior cingulate cortex (BA 32) and parts of right prefrontal cortex.

More detailed, we found significant activations bilaterally in SI during tickle (SS versus RS) and a contralateral activation in SI during anticipation (AS versus RS). Both complex finger movements and sensory vibratory stimulus yield a bilateral activation in SI (Burton, Videen, \& Raichle, 1993; Burton, Sinclair, \& Whang, 1998; Remy, Zilbovicius, Leroy-Willig, Syrota, \& Samson, 1994). This bilateral response may stem from callosal projections or cortico-cerebellar or corticospinal connectivity (Paulesu, Frackowiak, \& Bottini, 1997). The activation was more expressed in the SI during tickle than during anticipation (SS versus AS) and, as mentioned, the activation was bilateral during tickle while only unilateral during anticipation. The difference in bilaterality between SS and AS may, apart from sensitivity issues related to the applied method, also be due to the fact that the SS response handles a real bottom up sensory input, whereas the AS response represents a top down driven activation in SI. It is conceivable that the different origin of these responses influence whether the response is uni- or bilateral.

Contrary to the above, SI was not activated in the study by Pardo, Fox, and Raichle (1991) where a simple supra-threshold tactile sensory stimuli was used (von Frey's hair). The lack of response in SI, possibly due to lack of sensitivity, contrasted markedly to activations in the right prefrontal cortex and ventral premotor cortex (Pardo et al., 1991). In that study, the subjects were required to detect pauses in the touch and the authors interpreted the frontal activations as being part of a vigilance network. Ingvar (1985) discussed the phenomenon of extensive activity in the frontal cortex in response to sensory stimuli in terms of a "sensory motor paradox." Data presented by Paulesu et al. (1997), based on a paradigm where subjects received light sensory stimulation, also revealed a more expressed response in right prefrontal cortex as compared to primary sensory cortex. The location of the these frontal activations were inferior to those observed in the study of Pardo et al. (1991), but corresponded well to the prefrontal activations in our data and may support an interpretation in terms of the sensory motor paradox.

The SII region in humans is not yet well delineated, but has been proclaimed to be situated in BA 43 and 40 in the parietal opercular region, and these areas are bilaterally activated during processing of somatotactile stimulation (Coghill et al., 1994; Burton et al., 1993). There was a bilateral activation in the putative SII region in both main contrasts (see Table 1). In anticipation (AS versus RS) there was a marked side difference in that the activated region was much larger on the right side as compared to the left. This right-hemispheric dominance in activation may either be interpreted in terms of it being part of an attentional network or as lateralized processing of somatospatial information (Coull, 1998; Vallar, 1997; Kolb \& Whishaw, 1996; Hsieh et al., 1994).

There was an activation in the ACC both during anticipation (AS versus RS) and the sensory stimulation (SS versus RS). The level of activity in ACC is suggested to be related to various functions, among others supervisory control (Vogt, Finch, \& Olson, 1992). The expression of the attentional supervisory system can be an internal event without an overt behavioral expression (Norman \& Shallice, 1986). Hence, the ACC activation may be associated with the subject's intention to inhibit withdrawal, i.e., an expression of supervisory motor control of inhibiting movements. The activation in the ACC may also be considered as part of a spatial attention network or possibly as an affective response pertaining to the special nature of tickle (Nobre et al., 1997; Hsieh et al., 1994).

There were deactivations (i.e., decreases of activity) that were similar in the two contrasts (RS versus AS and RS versus SS) including left BA 8, sensorimotor cortex in areas located outside the area representing the foot and posterior cingulate cortex. The reason for the decreases of activity, i.e., registered concomitantly to increases in other areas has been debated. From a computational view it seems appropriate to inhibit task-irrelevant processing (Petersson, Elfgren, \& Ingvar, 1999; Ghatan et al., 1995, 1998; Shulman et al., 1997).

\section{Mechanisms of Anticipation}

Anticipation of an upcoming event includes deployment of appropriate networks of attention. These neural systems involve both a network subserving top-down modulation (sources of attention) and the sites of modulation in areas that (primarily and secondarily) process the attended information (Frith \& Dolan, 1997; Posner, 1994). The cortical correlates of the sources of attention have principally been assigned to the right posterior parietal cortex, the ACC, and the prefrontal cortex (Pardo et al., 1991; Mesulam, 1990; Posner \& Petersen, 1990). The sites of modulation are found, e.g., in the appropriate sensory areas as defined by the context, in our case the primary and secondary somatosensory cortices.

Attentional modulation of sensory association areas has been studied both in primates (Hyvarinen, Poranen, \& Jokinen, 1980) and in humans (Coull, 1998). Corbetta and colleges (1991) showed how neural activity was specifically enhanced in extrastriate areas in correlation to an attended visual feature by verbal instruction. The attention-driven enhancement of response was replicated in another study where radially moving dots constituted the visual stimulus. Motion sensitive areas in the visual cortex were activated in a 
contrast where the subjects were instructed to attend to the movement as compared to the situation when they were instructed to attend to another feature (Buchel et al., 1998). In this study, we demonstrate an increased activity in primary and secondary sensory cortices while anticipating a sensory stimulus supporting the concept of a top-down mechanism for the regulation of activity in attentional sites.

Top-down mechanisms of attention also induce decreases of activity in parts of the brain that do not pertain to the attended process (Shulman et al., 1997; Ghatan et al., 1995; Haxby et al., 1994). Ghatan et al. (1998) provided further evidence for the existence of top-down inhibition of irrelevant processing in an experiment where the subjects sequentially subtracted 7 from a given number with and without hearing irrelevant speech. There was a relatively lower activity in the primary auditory cortex during the arithmetical task and irrelevant speech condition as compared to the irrelevant speech alone. Decreased activity in response to an unattended feature has been shown to exist also within a modality (Rees, Frith, \& Lavie, 1997). When forcing the subject to perform a more in-depth analysis of a cognitive task, administered by visual input, perception and processing of moving dots (irrelevant stimuli) elicited less activity in visual area V5 in relative terms. The primary somatosensory cortex is somatotopically organized and hence it is possible to study differences in attentional modulation within this modality as well. The above mentioned decreases that were noted in our data set in the sensorimotor cortex in areas outside the target of stimulation may represent such an intra modal top down mechanism.

In a study by Drevets et al. (1995), where subjects anticipated either innocuous touches or painful stimuli, a deactivation was shown in SI surrounding the area representing the skin locus of both awaited stimuli and no change in SI area corresponding to the site of stimulation. The study of Drevets et al. was partly inspired by a study by Roland (1981) that used 2-D rCBF ${ }^{133}$ Xe-method. There, Roland reported an increase of the activity in an area tentatively defined as primary sensory cortex during anticipation of sensory touch (Roland, 1981). There were methodological differences between the studies of Drevets et al. (1995) and Roland (1981) that at least in part may explain the discrepant results. However, the present data provide support for both views, as we found increases in the part of SI that represented the target of the stimulus and decreases in other parts of the SI and adjacent parts of MI during the state of anticipation.

The SI and SII are parts of the network that may subserve the conscious experience of a sensory stimulus (SS versus RS). Anticipation in our paradigm imply an awaiting of a sensory input and this was coupled with increased neuronal activity in the contralateral primary sensory cortex as well as bilaterally in the secondary sensory cortex. There are several possible interpretations of this finding. One possibility is that the subjects during the anticipation of the expected tickle stimulus mentally imagine the skin sensation thereby causing a top-down generated activation in the sensory cortex. This interpretation would be in line with James' ideas of a co-localization of the functional expression of imagination and behavior (James, 1890). It would also be analogous to the conclusions of several studies of visual imagery where consciously driven imagery leads to activations in the lower order visual cortices (Kosslyn et al., 1997). A difference would be that a real visual experience and an imagined visual experience are described in very similar terms upon questioning the subject. Imagined touch, however, lacks many of the qualities of a real sensory percept when described by the subject. Our subjects did not describe a presensation of the touch during the anticipatory phase; they only communicated a sense of raised alertness. A more likely interpretation of the increased activity in SI and SII during anticipation would therefore be that the activations represent a tonic modulation of the relevant sensory areas in anticipating the sensory stimulus (Rees \& Frith, 1998). This explanation is also compatible with the finding of a decreased activity during AS outside the target area of the touch (Drevets et al., 1995).

The top-down modulation of the primary cortices can represent two separate mechanisms. It can be phasic in that it sets the stage for a more enhanced response to a stimulus via "gain setting." Alternatively, it may have a tonic character which implies a change in the base-line firing rate that gives a competitive advantage to a stimulus at an attended location (Rees \& Frith, 1998; Rees, Frackowiak, \& Frith, 1997). There is empirical evidence for both mechanisms (Rees \& Frith, 1998). Rees and Frith (1998) reported an experiment where visual or auditory stimulus rate was varied and the attended sensory channel was independently manipulated by a change of the instruction set. The finding was that the manipulation of the attention lead to signal changes that were independent of the change of rate of stimulus. In the present experiment, we have by decreasing the "rate of stimulation" to $0 \mathrm{~Hz}$ (during anticipation) been able to demonstrate a tonic up-regulation of the activity in the SI/SII and a concurrent down-regulation of the primary sensorimotor areas outside the area representing the foot. We interpret this as a tonic mechanism for the top-down modulation.

\section{CONCLUSIONS}

The present data indicate that anticipation of a sensory event is subserved by a neural network similar to that which subserves the processing of the actual sensory input. Further, this study indicates that activation of 
the primary sensory cortex can be obtained without intra-modal sensory input, i.e., the activity can be driven by top-down processes. From an information processing perspective, greater processing efficiency can be achieved by meeting the incoming information with what is already known or predicted, e.g., using model-based processing. The present paradigm may be seen as a demonstration of how extramodal contextual information can set the level of activity in a given neural system.

\section{MATERIAL AND METHODS}

Seven healthy subjects (four women and three men, right handed, nonsmoking, mean age 26 years, range 23-31 years) participated in the study. The subjects were selected to the study based on a preinterview and the fact that they displayed a moderate level of ticklishness. This level of ticklishness was chosen as a compromise considering both the ability to be still during the stimulation and the motivation to anticipate the stimulus. All subjects gave informed consent and the procedures were preapproved by the local ethical committee at the Karolinska Hospital.

\section{Experimental Design}

The experimental protocol was defined as a multisession blocked fMRI paradigm, each session consisting of three conditions. A visual stimulus consisting of a $20-\mathrm{cm}$ color square was displayed on a back projection screen. The square was either green or red and remained lit during the entire session. The subjects watched the screen from the supine position in the camera via a mirror. They were carefully instructed that they would not receive sensory stimulation as long as the square was green, while, after an unspecified time delay, they would be stimulated lightly on the sole of the right foot when the square had turned red. In all, there were the three conditions: Rest while fixating the green square (RS), anticipation of the sensory stimulation triggered by the red square (AS) and sensory stimulation plantarly while watching the red square (SS). The sensory stimulus was manually applied with a painter's brush at a frequency of 1-2 Hz. The size of the brush was about $.2 \mathrm{~cm}$ in width. In order to generate statistically valid data, each subject underwent repeated sessions including all three states of the paradigm. The subjects were scanned during four to six sessions, each lasting $3 \mathrm{~min}$. The paradigm was rather attention demanding and the multi-session protocol gave the subjects an opportunity to rest between sessions. To minimize physiological adaptation we restricted each period of sensory stimulation to 9-sec duration. The RS was always of 30-sec duration while AS was either of 9-sec duration, followed by 9 sec of SS, or of 18-sec duration followed by RS (see

\begin{tabular}{|ccccccc|c|c|}
\hline \multicolumn{7}{|c|}{ Time line for scan session } \\
\hline 2 & 10 & 6 & 10 & 6 & 10 & 6 & 10 \# images \\
\hline $30 \mathrm{~s}$ & $18 \mathrm{~s}$ & $30 \mathrm{~s}$ & $18 \mathrm{~s}$ & $30 \mathrm{~s}$ & $18 \mathrm{~s}$ & $30 \mathrm{~s}$ \\
\hline
\end{tabular}

Figure 3. Time line illustrating the principal green-red square sequence used in each 3-min session. The first two images (black) were discarded to eliminate magnetic saturation effects. Ten scans $(30 \mathrm{sec})$ were taken while the green square was showing as a reference condition (depicted in white). These were followed by six scans (18 $\mathrm{sec}$ ) with the red square (depicted in gray). During these six scans, the subject either anticipated during the whole $18 \mathrm{sec}$ or anticipated during $9 \mathrm{sec}$ and was sensory stimulated under the right foot during the following $9 \mathrm{sec}$.

Figure 3). In order to maintain high anticipatory responses the periods of AS where followed by SS in $66 \%$ of the occasions. The subjects were instructed not to move any part of the body, including the foot, throughout the sessions. After the last session they were asked to rate the mean sensation of tickle and the wish to move during the tickle on a visual analogue scale (VAS, 0-100).

\section{Data Acquisition}

Imaging was performed on a 1.5 T GE Signa Echospeed MR scanner with a standard circular head coil. An individually molded bite-bar was used to help subjects to prevent head movements. T1 weighted 3-D-SPGR images were acquired for the anatomical co-registration. The repetition time (TR), echo time (TE) and flip angle were $8 \mathrm{~ms}, 1.5 \mathrm{~ms}$ and $10^{\circ}$, respectively and the image resolution was $256 \times 256 \times 124$ with a voxel size of $.9 \times$ $.9 \times 1.8 \mathrm{~mm}$.

Functional images were obtained with a gradient echo-EPI sequence ( $\mathrm{TE}=70 \mathrm{msec}$, TR $=3 \mathrm{sec}$, and a $90^{\circ}$ flip angle). This represents a $\mathrm{T} 2 *$ weighted sequence, i.e., sensitive to the different magnetic properties of oxy- and deoxy-haemoglobin and allows determination of a blood oxygen level dependant (BOLD) contrast. The image volumes contained 13 horizontal 6-mm-thick slices positioned to cover the upper $7.8 \mathrm{~cm}$ of the brain. The in-plane resolution was set to $3 \times 3 \mathrm{~mm}$. Images were acquired sequentially. For each session, 60-image volumes were obtained. The shifts between the different conditions were synchronized with the data acquisition. To reduce magnetic saturation effects, each session began with two dummy scans that were discarded in the statistical analysis. Ten images (30 sec) were acquired while the green square was displayed (RS) alternated with six images (18 sec) with the red square. The block with the red square was either split in three images ( 9 sec) of AS followed by three images (9 sec) of SS or six scans $(18 \mathrm{sec})$ of AS and no SS. In each session, there 
were four blocks of green squares and three blocks of red squares stimulation (see Figure 3).

\section{Statistical Analysis}

Image preprocessing and statistical analysis were performed using SPM96 (Friston, Ashburner, et al., 1995; Friston, Holmes, et al., 1995). To adjust for head movement within and between the sessions all the volumes were realigned to a volume in the middle of the third or forth session (Friston, Williams, Howard, Frackowiak, \& Turner, 1996). Since correction was not expected to be greater than $1 \mathrm{~mm}$ and the TR was $3 \mathrm{sec}$, we used a simple first-order correction without "spin history" correction. No subject moved more than $1 \mathrm{~mm}$ in any direction in the time-series that were analyzed.

The morphological SPGR volume was spatially coregistered with a mean image created from the realigned EPI-BOLD images, taking into consideration that the image sets were differently weighted (i.e., T1 and T2, respectively). The morphological MRI was resliced to get the same voxel size as the EPI-BOLD images. The spatial normalization was performed on the resliced SPGR image to make it conform to the standard template of SPM96. The realigned functional images were then normalized and resliced using the parameters that had been determined from the SPGR image. To neutralize some of the inter-individual functional-anatomical residual, after spatial normalization, the functional data were spatially filtered with an isotropic 3-D gaussian smoothing kernel $(\mathrm{FWHM}=8$ $\mathrm{mm})$.

In addition, the data was temporally smoothed with a gaussian kernel of $6 \mathrm{sec}$ FWHM in order to estimate the effective degrees of freedom and apply the general linear model with ordinary least square estimation (Worsley \& Friston, 1995). To remove low frequency noise, a high pass filter with a periodicity of $96 \mathrm{sec}$ was included in the design matrix (Frackowiak, Fristeon, Frith, Dolan, \& Mazziotta, 1997; Friston, Holmes, et al., 1995). This time was chosen with regard to the cycle of rest $(30 \mathrm{sec})$ and activation $(9+9 \mathrm{sec})$ in order to limit the removal of signal of interest.

Using a delayed box-car wave form, comparisons of the different conditions (RS, AS, SS) were made by testing corresponding linear contrasts of parameters in the general linear model and evaluated on a voxel-byvoxel basis with SPM96, (Frackowiak et al., 1997; Friston, Holmes, et al., 1995; Worsley, Evans, Marrett, \& Neelin, 1992). The anatomical localization of the local maxima was determined in an approximate Talairach and Tournoux space (Talairach \& Tournoux, 1988). Cortical sites are referred to as Brodmann's areas (BA) as defined in the atlas of Talairach and Tournoux (1988) and in the Karolinska Computerized Brain Atlas of Greitz (Greitz, Bohm, Holte, \& Eriksson, 1991) (Table 1).

\section{Acknowledgments}

The research was supported by grants from The Swedish Medical Research Council (8246), the Swedish Bank Tercentennial foundation, The Family Ekman Foundation, Karolinska Institutet Foundations and the Knut and Alice Wallenberg Foundation. We are grateful to all supporting staff.

Reprint requests should be sent to Martin Ingvar, MD, PhD, Section of Clinical Neurophysiology, Department of Clinical Neuroscience, Karolinska Hospital, S-171 76 Stockholm, Sweden.

\section{REFERENCES}

Blakemore, S. J., Rees, G., \& Frith, C. D. (1998). How do we predict the consequences of our actions? A functional imaging study. Neuropsychologia, 36, 521-529.

Blakemore, S.-J., Wolpert, D. M., \& Frith, C. D. (1999). The cerebellum is involved in predicting the sensory consequences of action. Neuroimage, 9, S445.

Blakemore, S. J., Wolpert, D. M., \& Frith, C. D. (1998). Central cancellation of self-produced tickle sensation. Nature Neuroscience, 1, 635-640.

Broadbent, D. (1958). Perception and communication. Oxford: Pergamon.

Buchel, C., Josephs, O., Rees, G., Turner, R., Frith, C. D., \& Friston, K. J. (1998). The functional anatomy of attention to visual motion. A functional MRI study. Brain, 121, 1281-1294.

Burton, H., Sinclair, R. J., \& Whang, K. (1998). Vibrotactile stimulus order effects in somatosensory cortical areas of rhesus monkeys. Somatosensory and Motor Research, 15, 316-324.

Burton, H., Videen, T. O., \& Raichle, M. E. (1993). Tactilevibration-activated foci in insular and parietal-opercular cortex studied with positron emission tomography: Mapping the second somatosensory area in humans. Somatosensory and Motor Research, 10, 297-308.

Coghill, R. C., Talbot, J. D., Evans, A. C., Meyer, E., Gjedde, A., Bushnell, M. C., \& Duncan, G. H. (1994). Distributed processing of pain and vibration by the human brain. Journal of Neuroscience, 14, 4095-4108.

Corbetta, M., Miezin, F. M., Dobmeyer, S., Shulman, G. L., \& Petersen, S. E. (1991). Selective and divided attention during visual discriminations of shape, color, and speed: Functional anatomy by positron emission tomography. Journal of Neuroscience, 11, 2383-2402.

Coull, J. T. (1998). Neural correlates of attention and arousal: Insights from electrophysiology, functional neuroimaging and psychopharmacology. Progress in Neurobiology, 55, 343-361.

Drevets, W. C., Burton, H., Videen, T. O., Snyder, A. Z., Simpson, J. R., Jr., \& Raichle, M. E. (1995). Blood flow changes in human somatosensory cortex during anticipated stimulation. Nature, 373, 249-252.

Frackowiak, R., Friston, K., Frith, C., Dolan, R., \& Mazziotta, J. (1997). Human brain function. San Diego, CA: Academic Press.

Friston, K. J., Ashburner, J., Poline, J. B., Frith, C. D., Heather, J. D., \& Frackowiak, R. S. J. (1995). Spatial registration and normalization of images. Human Brain Mapping, 2, 165-189.

Friston, K. J., Holmes, A. P., Worsley, K. J., Poline, J. P., Frith, C. D., \& Frackowiak, R. S. J. (1995). Statistical parametric maps in functional imaging: A general linear approach. Human Brain Mapping, 2, 189-210. 
Friston, K. J., Williams, S. R., Howard, R., Frackowiak, R. S. J., \& Turner, R. (1996). Movement-related effects in fMRI time-series. Magnetic Resonance in Medicine, 35, 346-355.

Frith, C., \& Dolan, R. J. (1997). Brain mechanisms associated with top-down processes in perception. Philosophical Transactions of the Royal Society of London, Series B: Biological Sciences, 352, 1221-1230.

Gallistel, C. (1980). The organization of action: A new synthesis. Hillsdale, NJ: Erlbaum.

Ghatan, P. H., Hsieh, J. C., Petersson, K. M., Stone-Elander, S., \& Ingvar, M. (1998). Coexistence of attention-based facilitation and inhibition in the human cortex. Neuroimage, 7 , 23-29.

Ghatan, P. H., Hsieh, J. C., Wirsen-Meurling, A., Wredling, R., Eriksson, L., Stone-Elander, S., Levander, S., \& Ingvar, M. (1995). Brain activation induced by the perceptual maze test: A PET study of cognitive performance. Neuroimage, 2, $112-124$.

Greitz, T., Bohm, C., Holte, S., \& Eriksson, L. (1991). A computerized brain atlas: Construction, anatomical content and some applications. Journal of Computer Assisted Tomography, 15, 26-38.

Haxby, J. V., Horwitz, B., Ungerleider, L. G., Maisog, J. M., Pietrini, P., \& Grady, C. L. (1994). The functional organization of human extrastriate cortex: A PET-rCBF study of selective attention to faces and locations. Journal of Neuroscience, 14, 6336-6353.

Hodge, C. J., Jr., Dubroff, J. G., Huckins, S. C., \& Szeverenyi, N. M. (1998). Cortical activation evoked by somatosensory imagery determined by fMRI. NeuroImage, 7, S123.

Hsieh, J.-C. (1995). Central processing of pain; Functional brain imaging studies with PET. Stockholm: Thesis, Karolinska Institute.

Hsieh, J. C., Hagermark, O., Stahle-Backdahl, M., Ericson, K., Eriksson, L., Stone-Elander, S., \& Ingvar, M. (1994). Urge to scratch represented in the human cerebral cortex during itch. Journal of Neurophysiology, 72, 3004-3008.

Hsieh, J. C., Stone-Elander, S., \& Ingvar, M. (1999). Anticipatory coping of pain expressed in the human anterior cingulate cortex: A positron emission tomography study.

Neuroscience Letters, 262, 61-64.

Hyvarinen, J., Poranen, A., \& Jokinen, Y. (1980). Influence of attentive behavior on neuronal responses to vibration in primary somatosensory cortex of the monkey. Journal of Neurophysiology, 43, 870-882.

Ingvar, D. H. (1985). "Memory of the future": An essay on the temporal organization of conscious awareness. Human Neurobiology, 4, 127-136.

James, W. (1890). Principles of psychology. New York: Henry Holt.

Kolb, B., \& Whishaw, I. Q. (1996). Fundamentals of human neuropsychology, 4th ed. (pp. xii, 691). New York: W.H. Freeman.

Kosslyn, S. M., Thompson, W. L., \& Alpert, N. M. (1997). Neural systems shared by visual imagery and visual perception: A positron emission tomography study. Neuroimage, 6, 320-334.

Mesulam, M. M. (1990). Large-scale neurocognitive networks and distributed processing for attention, language, and memory. Annals of Neurology, 28, 597-613.

Mintz, T. (1967). Tickle-the itch that moves. A psychophysiological hypothesis. Psychosomatic Medicine, 29, 606-611.

Nobre, A. C., Sebestyen, G. N., Gitelman, D. R., Mesulam, M. M., Frackowiak, R. S., \& Frith, C. D. (1997). Functional localization of the system for visuospatial attention using positron emission tomography. Brain, 120, 515-533.
Norman, D. A., \& Shallice, T. (1986). Attention to action: Willed and automatic control of behavior. In R. J. Davidson, G. E. Schwarts \& D. Shapiro (Eds.), Consciousness and self-regulation. Advances in research and theory, Vol. 4 (pp. 1-18). New York: Plenum.

Pardo, J. V., Fox, P. T., \& Raichle, M. E. (1991). Localization of a human system for sustained attention by positron emission tomography. Nature, 349, 61-64.

Paulesu, R. S., Frackowiak, R. S. J., \& Bottini, G. (1997). Maps of somatosensory systems. In R. S. J. Frackowiak (Ed.), Human brain function (pp. xiii, 528). San Diego, CA: Academic Press.

Petersson, K. M., Elfgren, C., \& Ingvar, M. (1999). Dynamic changes in the functional anatomy of the human brain during recall of abstract designs related to practice. Neuropsychologia, 37, 567-587.

Posner, M. I. (1994). Attention: The mechanisms of consciousness. Proceedings of the National Academy of Sciences, U.S.A., 91, 7398-7403.

Posner, M. I., \& Petersen, S. E. (1990). The attention system of the human brain. Annual Review Neuroscience, 13, 25-42.

Rees, G., Frackowiak, R., \& Frith, C. (1997). Two modulatory effects of attention that mediate object categorization in human cortex. Science, 275, 835-838.

Rees, G., \& Frith, C. D. (1998). How do we select perceptions and actions? Human brain imaging studies. Philosophical Transactions of the Royal Society of London, Series B: Biological Sciences, 353, 1283-1293.

Rees, G., Frith, C. D., \& Lavie, N. (1997). Modulating irrelevant motion perception by varying attentional load in an unrelated task. Science, 278, 1616-1619.

Remy, P., Zilbovicius, M., Leroy-Willig, A., Syrota, A., \& Samson, Y. (1994). Movement- and task-related activations of motor cortical areas: A positron emission tomographic study. Annals of the Neurology, 36, 19-26.

Roland, P. E. (1981). Somatotopical tuning of postcentral gyrus during focal attention in man. A regional cerebral blood flow study. Journal of Neurophysiology, 46, 744-754.

Roth, M., Decety, J., Raybaudi, M., Massarelli, R., DelonMartin, C., Segebarth, C., Morand, S., Gemignani, A., Decorps, M., \& Jeannerod, M. (1996). Possible involvement of primary motor cortex in mentally simulated movement: A functional magnetic resonance imaging study. NeuroReport, 7, 1280-1284.

Rumelhart, D., \& McClelland, J. L. (1986). Parallel distributed processing: Explorations in the microstructures of cognition. Cambridge: MIT Press.

Schnieder, W., \& Shiffrin, R. (1977). Controlled and automatic human information processing: I. Detection, search, and attention. Psychology Review, 84, 1-66.

Shulman, G. L., Corbetta, M., Buckner, R. L., Raichle, M. E., Fiez, J. A., Miezin, F. M., \& Petersen, S. E. (1997). Topdown modulation of early sensory cortex. Cerebral Cortex, 7, 193-206.

Talairach, J., \& Tournoux, P. (1988). Co-planar stereotaxic atlas of the human brain. Stuttgart, Germany: George Thieme Verlag.

Vallar, G. (1997). Spatial frames of reference and somatosensory processing: A neuropsychological perspective. Philosophical Transactions of the Royal Society of London, Series B: Biological Science, 352, 1401-1409.

Vogt, B. A., Finch, D. M., \& Olson, C. R. (1992). Functional heterogeneity in cingulate cortex: The anterior executive and posterior evaluative regions. Cerebral Cortex, 2, 435443.

Von Holst, E. (1954). Relations between the central nervous system and the peripheral organs. The British Journal of Animal Behaviour, 2, 89-94. 
Weiller, C., Juptner, M., Fellows, S., Rijntjes, M., Leonhardt, G., Kiebel, S., Muller, S., Diener, H. C., \& Thilmann, A.F. (1996). Brain representation of active and passive movements. Neuroimage, 4, 105-110.

Wolpert, D. M. (1997). Computational approaches to motor control. Trends in Cognitive Sciences, 1, 209-216.
Worsley, K. J., Evans, A. C., Marrett, S., \& Neelin, P. (1992). A three-dimensional statistical analysis for CBF activation studies in human brain. Journal of Cerebral Blood Flow Metabolism, 12, 900-918.

Worsley, K. J., \& Friston, K. J. (1995). Analysis of fMRI time-series revisited-again. Neuroimage, 2, 173-181. 